\title{
<arttitle> Differential stress induced by thiol adsorption on facetted nanocrystals
}

$<$ aug> Moyu Watari ${ }^{1}$, Rachel A. McKendry ${ }^{1}$, Manuel Vögtli ${ }^{1}$, Gabriel Aeppli ${ }^{1}$, Yeong-Ah Soh ${ }^{2}$, Xiaowen Shi ${ }^{1}$, Gang Xiong ${ }^{1}$, Xiaojing Huang ${ }^{1}$, Ross Harder ${ }^{3}$ \& Ian K. Robinson ${ }^{1}$

$<$ aff $>{ }^{1}$ London Centre for Nanotechnology, University College, London, WC1E 6BT, $U K$

$<$ aff $>{ }^{2}$ London Centre for Nanotechnology, Imperial College, London, SW7 2AZ, UK

$<$ aff $>{ }^{3}$ Advanced Photon Source, Argonne, IL 60439, USA

$<$ footnote $>$ Style tag for any footnotes linked to authors (e.g. Present address: Department of ...).

$<$ abs $>$ Polycrystalline gold films coated with thiol-based self assembled monolayers (SAM) form the basis of a wide range of nanomechanical sensor platforms [1]. The detection of adsorbates with such devices relies on the transmission of mechanical forces, which is mediated by chemically derived stress at the organicinorganic interface. Here, we show that the structure of a single $300 \mathrm{~nm}$-diameter facetted gold nanocrystal, measured with coherent $X$-ray diffraction, changes profoundly after the adsorption of one of the simplest SAM-forming organic molecules. On self-assembly of propane thiol, the crystal's flat facets contract radially inwards relative to its spherical regions. Finite-element modelling indicates that this geometry change requires large stresses which are comparable with those observed in cantilever measurements. The large magnitude and slow kinetics of the contraction can be explained by an intermixed gold-sulphur layer which has recently been identified crystallographically [2]. Our results illustrate the importance of crystal edges and grain boundaries in interface chemistry and 


\section{have broad implications for the application of thiol-based SAMs, ranging from nanomechanical sensors to coating technologies.}

$<\mathrm{p}>$ The especially strong bond that forms between sulphur and gold is the basis for numerous self-assembled metal-organic devices. Gold is a noble metal towards almost all environmental species with the exception of sulphydryls, such as thiols, which readily form monolayer coatings of high stability. Biomedical sensors[1] generally rely on some physical contact between biomolecules in an extracellular environment with some inorganic readout system, for example silicon-based electronic circuitry[3]. In particular, thiol end groups linked to biomolecular agents forming selfassembled monolayers (SAMs)[4-6] on one surface of a sensitive cantilever can be used as a chemo-mechanical sensor $[1,7,8]$. To engineer sensors with high sensitivity and reliability[3,7-9], it is vital to understand the fundamental nature of the communication mechanism between the forces generated by binding interactions at the surface/solution interface with the ultimate macroscopic deflection of the cantilever.

$<\mathrm{p}>$ Gold nanoparticles have attracted considerable interest because of their diverse applications. Central to the success of virtually all applications is the need to tailor the nanoparticles with organic coatings, often SAMs, which impart both stability and specific functionality[4-6]. While much effort has focused on optimising the activity of the immobilised layer, for example antibodies or enzymes[7], surprisingly little is understood about the SAM's influence on the underlying metallic nanoparticle itself. In this letter, we exploit the sensitivity of coherent X-ray diffraction to quantify the strains induced by thiols on a gold nanocrystal. The substantial stress forces found are comparable with those observed in cantilever adsorption experiments.

$<\mathrm{p}>$ The structure of the sulphur-gold interface is surprisingly complex. Classical surface-science studies of sulphur layers on single crystal gold surfaces[10] have found 
remarkably few well-ordered structures, and even those are marginally stable. The most stable $\mathrm{Au}(111)$ surface has an incommensurate 'herringbone' reconstruction that shows significant compressibility leading to the expulsion of 'adatoms'[11]. The ejected $\mathrm{Au}$ atoms have a tendency to form linear S-Au-S 'staple' arrangements decorating otherwise close-packed surfaces[10,12,13]. The interplay of reconstruction and adsorption favours thiol attachment to disordered gold surfaces, either facetted high-index crystal directions, or the inherently stepped surfaces of small gold grains. Indeed, a recent crystallographic study[2] of single-phase $\mathrm{Au}_{102} \mathrm{RS}_{44}$ gold nanoparticle-thiol clusters revealed a decahedral crystalline core particle coated with a shell $0.25 \mathrm{~nm}$ thick with enlarged $\mathrm{Au}-\mathrm{Au}$ spacings and interpenetration of the thiol ligand species. Far from having a well-defined boundary between the metal and the organic sides of the interface, this unusual complex was found to contain a mixed compound layer as its equilibrium configuration[2].

$<\mathrm{p}>$ We employ the new method of synchrotron-based coherent X-ray diffraction (CXD) to compare three-dimensional (3D) images of individual gold nanocrystals before and after coating with thiols. As we have shown previously, CXD is highly sensitive to internal strains within a crystal[14], which appear as a change of phase in the complex image. The electron density of the sample appears as the amplitude of the image while its phase $\varphi(\mathbf{r})$ represents the projection of the displacement from the ideal crystal lattice, $\mathbf{u}(\mathbf{r})$, onto the reciprocal lattice vector, $\mathbf{G}$, of the Bragg peak used for the diffraction measurement, $\boldsymbol{\varphi}(\mathbf{r})=\mathbf{G} \bullet \mathbf{u}(\mathbf{r})$ [15]. In recent work[16], multiple G-vectors have allowed a full mapping of the strain tensor within a nanocrystal. Here we use a single (11-1) G-vector to image the component of the lattice displacements perpendicular to nanocrystal facets pointing in that direction.

$<\mathrm{p}>$ Fig 1 shows the distinct changes seen directly in the CXD pattern of a gold nanocrystal upon adsorption of the propane thiol, transported through the vapour phase 
from a drop of ethanol on a remotely actuated syringe, as described in the SI. Repeated measurements showed the transfomation was reproducible, gradual but irreversible. The data were phased and inverted using standard methods[15], described in the SI, to the resulting complex image in Fig. 2a, whose amplitude shows the facetted equilibrium crystal shape (ECS; see SI)[17]. The 3D image measures $310 \mathrm{~nm}$ across at the widest point. The facets can be seen to line up with the $\{111\}$ crystal directions shown. The real-space phase, $\varphi(\mathbf{r})$, used to colour the density isosurface shown, indicates the presence of some residual strains, presumably due to differential thermal expansion during the sample preparation[14]. The magnitude of the strains is considerably smaller than the $\pm \pi / 2$ range (corresponding to displacement by one quarter of a lattice spacing) allowed by the phasing algorithm [18]. Both the flattened overall morphology, with enlarged (111) and (-1-1-1) facets along the surface normal, and the residual strains are an indication that the true equilibrium shape[17] has not been reached by the sample preparation, dewetting a $20 \mathrm{~nm}$ film from a Si wafer substrate (see methods section). Nevertheless images of a single nanocrystal, free from major defects and isolated from its neighbours on the substrate, are clearly achieved.

$<\mathrm{p}>$ The "Difference Map" (DM) method is widely employed in protein crystallography to identify localized small changes, for example in the active sites of enzymes[19]. The DM makes the approximation that the reciprocal-space phases of the diffraction pattern are the same before and after a small change is made in the structure. This approximation is particularly good when the small changes are localized in a small region of real space, so will therefore be widely distributed in reciprocal space[19]. It is clear from the small intensity differences in Fig. 1 that the DM approximation is appropriate, so long as the data are correctly centred, as discussed in the supplementary information (SI). The resulting DM density, $\Delta \rho(\mathbf{r})$, is the difference of the two complex density functions, given by the Fourier transform of the differences between the CXD 
patterns, using the phased diffraction of one of them as the common phase function, $\alpha(\mathbf{q})$ :

$<\mathrm{fd}>\Delta \rho(\mathbf{r})=\rho_{2}(\mathbf{r})-\rho_{1}(\mathbf{r})=\int\left[\sqrt{ }_{2}(\mathbf{q})-\sqrt{ }_{1}(\mathbf{q})\right] \mathrm{e}^{\mathrm{i} \alpha(\mathbf{q})} \mathrm{e}^{\mathrm{i} \mathbf{q} \cdot \mathbf{r}} \mathrm{d}^{3} \mathbf{q}$

$<$ p-ni $>$ where $\mathbf{q}=\mathbf{Q}-\mathbf{G}$ represents the deviation of the momentum transfer, $\mathbf{Q}$, from the reciprocal lattice vector, $\mathbf{G}$, of the Bragg peak used. The resulting complex function, $\Delta \rho(\mathbf{r})$, is an image of the changes before and after the sample was modified. Changes of crystal strain, when the shape and density are constant, should appear mainly in the imaginary part of $\Delta \rho(\mathbf{r})$.

$<\mathrm{p}>$ Difference maps, directly measuring the changes in the crystal strain due to thiol adsorption, are shown in Figs. $2 \mathrm{~b}$ and $2 \mathrm{c}$ for the two data sets of the dosed sample from Fig. 1 (centre and right), after subtracting the undosed data (left), using the phases $\alpha(\mathbf{q})$ obtained for the latter. A contour map of the DM amplitude slices through a transparent ghost image of the crystal density itself. Perpendicular cuts, both containing the measurement (11-1) G-vector, are shown for both data sets. There are two clear lobes of difference, which are seen to align with G. In Fig. 3a, the lobes have been coloured according to their phases, light blue being close to $-\pi / 2$ in the backwards (-1-11) direction, and yellow near $\pi / 2$ in the forward (11-1), confirming that the complex $\Delta \rho(\mathbf{r})$ is imaginary. On the crystal, these opposing displacements are therefore both directed radially inwards. The lobes lie close to the surface, suggesting they correspond to surface strains, since they decay into the bulk over a length scale comparable with the facet feature size[20]. The maximum value of $|\Delta \rho(\mathbf{r})|$ is $50 \pm 20$ units in Fig. $2 \mathrm{~b}$ on a scale on which the density $\rho(\mathbf{r})$ is 200 units, so the phase rotation between $\rho_{1}(\mathbf{r})$ and $\rho_{2}(\mathbf{r})$ is $0.25 \mathrm{rad}$ and $|\mathbf{u}(\mathbf{r})|=0.009 \mathrm{~nm}$. For the second measurement in Fig $2 \mathrm{c}, \Delta \rho(\mathrm{r})$ is $100 \pm 30$ units giving $0.5 \mathrm{rad}$ and $|\mathbf{u}(\mathbf{r})|=0.019 \mathrm{~nm}$. Relatively few other 
features are seen in the DM: any radial strains on the other six $\{111\}$ facets, lying $71^{\circ}$ away from $\mathbf{G}$, are invisible as they do not contribute much to $\varphi(\mathbf{r})=\mathbf{G} \cdot \mathbf{u}(\mathbf{r})$.

$<\mathrm{p}>$ The pattern of displacement observed within the crystal upon thiol coating of its surface can be attributed to differential surface stress. In Fig. 3b, a Finite Element Analysis (FEA) of the strain in a model Au nanocrystal shows components of $\mathbf{u}(\mathbf{r})$ calculated parallel and perpendicular to the facets. To model the differential effects, we applied a stress $\sigma_{\mathrm{S}}$ on the $\{111\}$ facets and left the spherical surface regions in between unstressed. Positive (tensile) stress on the facets produced the observed inward displacements, following the standard sign convention (see SI). The displacement is mostly in the direction perpendicular to the facet, even though the surface stress acts laterally. This supports the argument made above that the strains on the six facets not oriented along the (11-1) $\mathbf{G}$-vector contribute less to the measured phase, $\varphi(\mathbf{r})=\mathbf{G} \bullet \mathbf{u}(\mathbf{r})$. Scaling the FEA calculation to the observed experimental net displacement above, the stress difference between the facets and spherical regions was determined to be $\Delta \sigma_{\mathrm{S}}=4.5 \pm 2 \mathrm{~N} / \mathrm{m}$ for the first measurement (one hour exposure) increasing to $\Delta \sigma_{\mathrm{S}}=9.5 \pm 3 \mathrm{~N} / \mathrm{m}$ for the second (two hours). Two more FEA calculations, with $\sigma_{\mathrm{S}} / 2$ (facet) and $-\sigma_{\mathrm{S}} / 2$ (spherical) surface regions in Fig. $3 \mathrm{c}$, and with 0 (facet) and $-\sigma_{\mathrm{S}}$ (spherical) in Fig. 3d, give almost identical patterns of displacements. The strain pattern is therefore largely insensitive to the total net stress, which could be positive or negative, but depends only on the difference: we conclude that the spherical regions are more compressively stressed than the facets by an amount $\Delta \sigma_{\mathrm{S}}$.

$<\mathrm{p}>$ In cantilever bending experiments, chemical changes within one-sided coatings cause stresses leading to the deflections measured[21,22]. A compressive surface stress change of $\Delta \sigma_{S}=-0.25 \mathrm{~N} / \mathrm{m}$, due to formation of a SAM, was originally reported for a cantilever coated by vapour diffusion in air, similar to our experiments[23]. More recently, Godin et al found $\Delta \sigma_{\mathrm{S}}=-0.5 \mathrm{~N} / \mathrm{m}$ for a $90 \mathrm{~nm}$-grained 
Au film and as much as $-16 \mathrm{~N} / \mathrm{m}$ for a $600 \mathrm{~nm}$-grained film[24], spanning the range seen in our experiments. These represent macroscopic long-range averages for an evaporated film disregarding morphology and connectivity effects. Berger et. al. reported a linear chain-length dependence[23] for longer-chain thiols $\left(\mathrm{C}_{4}\right.$ to $\left.\mathrm{C}_{14}\right)$ while Godin et. al. reported almost none for $\mathrm{C}_{6}$ to $\mathrm{C}_{10}$ [25]. Both studies found always the same compressive direction; however we find the opposite, tensile bending with a stress of $\Delta \sigma_{\mathrm{S}}=+0.15 \mathrm{~N} / \mathrm{m}$ for propane thiol $\left(\mathrm{C}_{3}\right)$ in our own cantilever bending measurements, described in the SI. The tensile stress reported here is consistent with our previous unpublished experimental work and attributed to the strong surface interactions but weaker chain-chain interactions for short $\mathrm{C}_{3}$ thiols, compared with the longer chain thiols typically studied by other researchers. The long time constants found in all studies[22-24], up to $10 \mathrm{~h}$ in one case[24], were explained in terms of structural rearrangements within the SAM. In our X-ray measurement of the surface of a nanocrystal, a uniform surface stress would cause only an overall change of lattice constant; our observation of a clear pattern of differential surface strain informs us that the stress varies across the surface.

$<\mathrm{p}>$ Our discovery is that the stress generated by thiol adsorption on gold has a fundamentally different nature in the curved, nominally spherical, regions of the crystal surface than its flat facets, leading to the clear pattern of strain we observe. The magnitudes of surface stress involved, up to $-16 \mathrm{~N} / \mathrm{m}$ seen for cantilevers[24] and the $9.5 \mathrm{~N} / \mathrm{m}$ differential stress reported here, are large for clean metals which typically have tensile surface stresses in the range of $4 \mathrm{~N} / \mathrm{m}$ [26]. Chemisorption of electronegative elements on metals typically leads to compressive stress changes in the range of $-5 \mathrm{~N} / \mathrm{m}$ [27]. Much of the discussion of SAMs in the literature[23-25] considers only the flat configuration appropriate to surface science studies on extended crystals[10] but, as pointed out[25], even for long-chain thiols, the large stress cannot arise from the van der Waals chain-chain interactions or other weak forces alone, but requires at least ionic or 
covalent rearrangements. Indeed the Au-S interaction plays a crucial role in SAM formation: the structure of very small $(1.6 \mathrm{~nm})$ thiolated nanocrystals, reported by Jadzinsky et al[2], has its Au-Au spacings strongly disrupted and sulphur intermixed in with gold in the outer layers. Our findings support this model and show strong thiolinduced deformations of our $300 \mathrm{~nm}$ crystals with strains penetrating more than $20 \mathrm{~nm}$ from the outer surface towards the crystal core, where strains are absent. The tightradius spherical parts of our $300 \mathrm{~nm}$ nanocrystals might also undergo strong Au-S intermixing which would indeed be able to provide sufficient stress. Intermixing reactions involving atomic diffusion of $\mathrm{Au}$ at room temperature, would also be an attractive explanation of the relatively slow kinetics seen in both our X-ray and the cantilever experiments.

$<\mathrm{p}>$ Our observation of relative contraction of the facets and expansion of the curved surface regions of Au nanocrystals, illustrated in Fig 3(e), leads to the conclusion that the curved and flat regions react very differently to SAM-forming thiol ligands. This explains the strong effect of grain size on cantilever bending stresses in polycrystalline films[24]. To the extent that surface stress and surface energy are coupled[26], we would expect the presence of thiol ligands to affect the facet/sphere proportion in the ECS[17]. Variation of growth morphology, also directly influenced by stress[11,29], might explain how surface-active thiolated 'additives' can lead to 'Curvature Enhanced Acceleration' in the damascene electroplating process[30].

\section{$<$ meth1ttl $>$ Methods}

\section{$<$ meth1hd $>$ Gold Nanocrystals}

$<$ meth1 $>$ Silicon wafers were first cleaned in Pirahna solution and then coated with an evaporated layer of $20 \mathrm{~nm}$ gold on top of an evaporated $1 \mathrm{~nm}$ Ti adhesion layer. The thin-film samples were then heated to $1050^{\circ} \mathrm{C}$ for 12 hours in a lab furnace purged with 
flowing nitrogen gas. Subsequent Scanning Electron Microscopy (SEM) showed the formation of arrays of nanocrystals with a small range of sizes centred around $200 \mathrm{~nm}$ in diameter, separated by $1-2 \mu \mathrm{m}$. SEM showed they had not quite reached the expected ECS, spherical with $\{111\}$ facets; due to incomplete dewetting, the crystals were wider than they were tall with the specular (111) facet more extended than the six off-specular side facets. The aspect ratio was about 1.5:1. Importantly for this experiment, both rough, spherical and flat, facetted surface regions were simultaneously present in the shape.

$<$ meth 1 hd $>$ CXD experiments

$<$ meth $1>$ Coherent X-rays of $8.92 \mathrm{keV}$ from the 34-ID-C beamline of the Advanced Photon Source (APS) were focussed onto the sample using Kirkpatrick-Baez (KB) mirrors which achieved a focus around $2 \times 2 \mu \mathrm{m}^{2}$. The diffraction pattern was measured using a direct-detection charge-coupled device (CCD) with $22.5 \mu \mathrm{m}^{2}$ pixels located on the detector arm, $1.03 \mathrm{~m}$ away from the sample. The 3D CXD pattern was then acquired as a $\theta$-scan, rotating in 81 steps of $0.01^{\circ}$ about a vertical axis. The CXD patterns were inverted to images with a 3D Fourier transform and coordinate transformation, following support-based phasing using a version of Fienup's Hybrid Input-Output (HIO) algorithm[14,15,28].

$<$ meth 1 hd $>$ Thiol dosing

$<$ meth1 $>$ A syringe with its needle piercing the nitrogen-gas environment above the sample, was remotely actuated to avoid any disturbance of the KB-sample alignment at the submicron level. The syringe was previously filled with a $5 \mathrm{mM}$ solution of propane thiol, $\mathrm{C}_{3} \mathrm{H}_{7} \mathrm{SH}$, dissolved in pure ethanol.

$<$ meth 1 hd $>$ Finite-element analysis (FEA) 
$<$ meth $1>$ The COMSOL FEA package was used to simulate the strain pattern introduced in a facetted gold sphere of $300 \mathrm{~nm}$ diameter by a differential surface stress. Four circular flat facets, each subtending $40^{\circ}$, were placed in contact with a thin skin of the same material of thickness $\mathrm{h}=5 \mathrm{~nm}$ containing one value of the bulk isotropic stress of $\sigma_{\mathrm{x}}=\sigma_{\mathrm{y}}=\sigma_{\mathrm{z}}=\sigma_{\mathrm{B}}$ (in Pa units) over the facets and another value over the spherical surface regions. The surface stress, equal to the "mechanical surface tension", is then given by $\sigma_{\mathrm{S}}=\sigma_{\mathrm{B}} \mathrm{h}$ in units of $\mathrm{N} / \mathrm{m}$ or $\mathrm{J} / \mathrm{m}^{2}$.

$<$ received $>$ Style tag for received and accepted dates (omit if these are unknown).

$<$ bibcit $>1$. "Nanomechanical detection of antibiotic mucopeptide binding in a model for superbug drug resistance", J. W. Ndieyira, M. Watari, A. D. Barrera, D. Zhou, M. Vögtli, M. Batchelor, M. A. Cooper, T. Strunz, M. A. Horton, C. Abell, T. Rayment, G. Aeppli \& R. A. McKendry, Nature Nanotechnology 3 691-696 (2008)

$<$ bibcit $>2$. "Structure of a thiol monolayer-protected gold nanoparticle at 1.1 angstrom resolution” P. D. Jadzinsky, G. Calero, C. J. Ackerson, D. A. Bushnell and R. D. Kornberg, Science 318 430-3 (2007)

$<$ bibcit>3. "Cantilever-like micromechanical sensors", A. Boisen, S. Dohn, S. S. Keller, S. Schmid and M. Tenje, Rep. Prog. Phys. 74036101 (2011) $<$ bibcit $>4$. "Adsorption of bifunctional organic disulfides on gold surfaces", R. G. Nuzzo and D. L. Allara, J. Am. Chem. Soc. 1054481 (1983)

$<$ bibcit $>5$. "Formation of monolayer films by the spontaneous assembly of organic thiols from solution onto gold", C. D. Bain, E. B. Troughton, Y. T. Tao, J. Evall, G. M. Whitesides \& R. G. Nuzzo, J. Am. Chem. Soc. 111321 (1989)

$<$ bibcit $>$ 6. "Self-Assembled Monolayers of Thiolates on Metals as a Form of Nanotechnology”, J. C. Love, L. A. Estroff, J. K. Kriebel, R. G. Nuzzo \& G M. Whitesides, Chem. Rev. 1051103 (2005) 
<bibcit> 7. "Translating Biomolecular Recognition into Nanomechanics," J. Fritz, M.K. Baller, H.P. Lang, H. Rothuizen, P. Vettiger, E. Meyer, H.-J. Guntherodt, Ch. Gerber \& J.K. Gimzewski, Science 288 316-318 (2000)

$<$ bibcit $>$ 8. "Multiple label-free biodetection and quantitative DNA binding assays on a nanomechanical cantilever array”, R. A. McKendry, J. Zhang, Y. Arntz, T. Strunz, M. Hegner, H-P. Lang, M.K. Baller, U. Certa, H-J. Guntherodt, \& C. Gerber, Proc. Natl. Acad Sci. U.S.A. 999783 (2002)

$<$ bibcit $>$ 9. "Creating Favorable Geometries for Directing Organic Photoreactions in Alkanethiolate Monolayers”, M. Kim, J. N. Hohman, Y. Cao, K. N. Houk, H. Ma, A. K.-Y. Jen \& P. S. Weiss, Science 3311312 (2011)

$<$ bibcit> 10. "Gold adatom as a key structural component in self-assembled monolayers of organosulfur molecules on $\mathrm{Au}(111)$ " P. Maksymovych, O. Voznyy, D. B.

Dougherty, D. C. Sorescu, J. T. Yates, Progress in Surface Science 85206 (2010)

$<$ bibcit> 11. "Electrocompression of the $\mathrm{Au}(111)$ Surface Layer during $\mathrm{Au}$

Electrodeposition”, A. H. Ayyad, J. Stettner, and O. M. Magnussen, Phys. Rev. Lett. 94, $066106(2005)$

$<$ bibcit $>12$. "True Nature of an Archetypal Self-Assembly System: Mobile Au-Thiolate Species on Au(111)”, Miao Yu, N. Bovet, C. J. Satterley, S. Bengio, K. Lovelock, P. K. Milligan, R. G. Jones, D. P. Woodruff, and V. Dhanak, Phys. Rev. Lett 97, 166102 (2006)

<bibcit> 13. "The Staple Motif: A Key to Stability of Thiolate-Protected Gold Nanoclusters”, D. Jiang, M. L. Tiago, W. Luo, and S. Dai, J. Am. Chem. Soc., 130, 2777 (2008) 
$<$ bibcit> 14. "Three-dimensional Mapping of a Deformation Field inside a Nanocrystal”, M. A. Pfeifer, G. J. Williams, I. A. Vartanyants, R. Harder, and I. K. Robinson, Nature 442, 63 (2006)

$<$ bibcit $>15$. "Coherent Diffraction Imaging of Strains on the Nanoscale", I. K.

Robinson and R. Harder, Nature Materials 8 291-298 (2009)

$<$ bibcit $>16$. "Three-dimensional imaging of strain in a single $\mathrm{ZnO}$ nanorod" M. C.

Newton, S. J. Leake, R. Harder and I. K. Robinson, Nature Materials 9 120-4 (2010)

$<$ bibcit> 17. M. Wortis, in "Chemistry and Physics of Solid Surfaces VII," Springer

Series in Surface Science 10, ed. R. Vanselow and R. Howe, 367 (1988)

$<$ bibcit $>18$. "Phase retrieval from the magnitude of the Fourier transforms of nonperiodic objects”, J. Miao, D. Sayre and H. N. Chapman, J. Opt. Soc. America 15 1662 (1998)

<bibcit> 19. "The difference Fourier technique in protein crystallography: errors and their treatment", R. Henderson and J. K. Moffat, Acta Cryst. B27, 1414-20 (1971) $<$ bibcit> 20. "Orientation variation of surface strain", R. Harder, M. A. Pfeifer, G. J. Williams, I. A. Vartaniants and I. K. Robinson, Physical Review B 76115425 (2007) $<$ bibcit> 21. "Physics of Nanomechanical Biosensing on Cantilever Arrays", M. L. Sushko, J. H. Harding, A. L. Shluger, R. A. McKendry, M. Watari, Advanced Materials $203848(2008)$

$<$ bibcit $>22$. "Investigating the molecular mechanisms of in-plane mechanochemistry on cantilever arrays", M. Watari, J. Galbraith, H. P. Lang, M. Sousa, M. Hegner, C. Gerber, M. A. Horton and R. A. McKendry, J. Am. Chem. Soc. 129 601-9 (2007) $<$ bibcit $>23$. "Surface Stress in the Self-Assembly of Alkanethiols on Gold", R. Berger, E. Delamarche, H-P. Lang, C. Gerber, J. K. Gimzewski, E. Meyer, H-J. Guentherodt, Science 276 2021-4 (1997) 
$<$ bibcit> 24. "Surface Stress, Kinetics, and Structure of Alkanethiol Self-Assembled Monolayers" M. Godin, P. J. Williams, V. Tabard-Cossa, O. Laroche, L. Y. Beaulieu, R. B. Lennox and P. Grutter, Langmuir 20 7090-6 (2004)

$<$ bibcit $>25$. "Cantilever-based sensing: the origin of surface stress and optimization strategies”, M. Godin, V. Tabard-Cossa, Y. Miyahara, T. Monga, P. J. Williams, L. Y. Beaulieu, R. B. Lennox and P. Grutter, Nanotechnology 21075501 (2010) $<$ bibcit> 26. "Surface stress of clean and adsorbate-covered solids", W. Haiss, Rep. Prog. Phys. 64 591-648 (2001)

$<$ bibcit $>27$. "The role of surface stress in reconstruction, epitaxial growth and stabilization of mesoscopic structures”, H. Ibach, Surf. Sci. Rep. 29 195-263 (1997) <bibcit> 28. "Phase retrieval algorithms: a comparison", J. R. Fienup, Appl. Opt. 21, 2758-69 (1982)

$<$ bibcit> 29. "Kinetic evolution and equilibrium morphology of strained islands", A. Rastelli, M. Stoffel, J. Tersoff, G. S. Kar and O. G. Schmidt, Phys. Rev. Lett. 95026103 (2005)

$<$ bibcit $>30$. "The chemistry of additives in damascene copper plating", P. M. Vereecken, R. A. Binstead, H. Deligianni and P. C. Andricacos, IBM J. Res. \& Dev., 49 (2005)

$<$ supp $>$ Supplementary Information accompanies the paper on www.nature.com/nature.

$<$ ack> Acknowledgements: work supported by the "nanosculpture" Advanced Grant from the European Research Council, a Science and Innovation Award for Nanometrology and a Nanotechnology "Grand Challenge in Healthcare” from the UK Engineering and Physical Sciences Research Council. Measurements were performed at APS, which is operated by the US Department of Energy.

<ack> Authors' Contributions: Project was conceived by MW, RAM, GA, YS \& IKR. MW, RH \& IKR performed the X-ray measurements, MW \& MV the cantilever measurements, XS \& GX the FEA 
analysis and MW, XH, RH \& IKR the difference map analysis. All authors contributed to interpreting the results and writing the paper.

$<$ corr $>$ Correspondence and requests for materials should be addressed to IKR. (i.robinson@ucl.ac.uk)

$<$ LEGEND> Figure 1. Measured coherent X-ray diffraction patterns of a gold nanocrystal before (left), after vapour-phase thiol dosing (centre) and one hour later (right). The interference fringes are due to the finite extent of the nanocrystal. The top row shows the central frame of the diffraction pattern, directly on the (11-1) Bragg peak, enlarged to show the centre. The bottom row shows the same patterns on the same scale, offset by five frames around the horizontal $\theta$-axis, or $0.05^{\circ}$ on the rocking curve. The colour scale is calibrated in units of X-ray photons per pixel; the centre of the pattern reaches a value of 9000 , but is saturated here.

$<$ LEGEND> Figure 2. Difference Map analysis showing the location of the crystal distortions due to thiol binding. (a) Density isosurface views of the derived shape of the crystal investigated in this paper. The rods point along the $\{111\}$ crystal directions, along which facets can be clearly seen, with the biggest along (111), the substrate surface normal. The measurements were made along $\mathrm{G}=(11-1)$ indicated by the arrow. The contour has been coloured by the local value of the phase, indicating the presence of a strain that is intrinsic to the crystal before dosing. (b) Contour map of sections of the difference maps calculated between the data set after dosing with thiol for one hour (Fig. 1 centre) and the undosed data (Fig. 1 left). (c) Same after two hours dosing. Two perpendicular cross sections are shown, both containing the (11-1) Gvector, both passing through the centre of the crystal. Only the magnitude of difference density is shown on a relative colour scale running from blue to red in each panel. 
$<$ LEGEND> Figure 3. Measured strain and finite-element analysis (FEA) simulation of the effect of surface stress. (a) Image of the measured crystal with a single contour of the difference density $\Delta \rho(\mathbf{r})$ superimposed, coloured according to its phase. (b) Calculated vertical component of the displacements of a model facetted gold nanocrystal with a tensile surface stress $\sigma_{S}=1.5 \mathrm{~N} / \mathrm{m}$ applied to the facets alone. (c) Same with a tensile surface stress $\sigma_{S}=0.75 \mathrm{~N} / \mathrm{m}$ applied to the facets and a compressive surface stress $\sigma_{S}=-0.75 \mathrm{~N} / \mathrm{m}$ applied to the spherical surface regions. (d) Same with a compressive surface stress $\sigma_{\mathrm{S}}=-1.5 \mathrm{~N} / \mathrm{m}$ applied to the spherical surface regions alone. A sphere of radius $145 \mathrm{~nm}$ is attached to a skin layer of $5 \mathrm{~nm}$ thickness used to apply the stress. Four $40^{\circ}$ facets intercept the sphere, also with $5 \mathrm{~nm}$ skins. (e) Cartoon representation of the relative motions at the nanocrystal surface induced by thiol adsorption: the crystal's flat facets are observed to contract inwards relative to its spherical regions. 


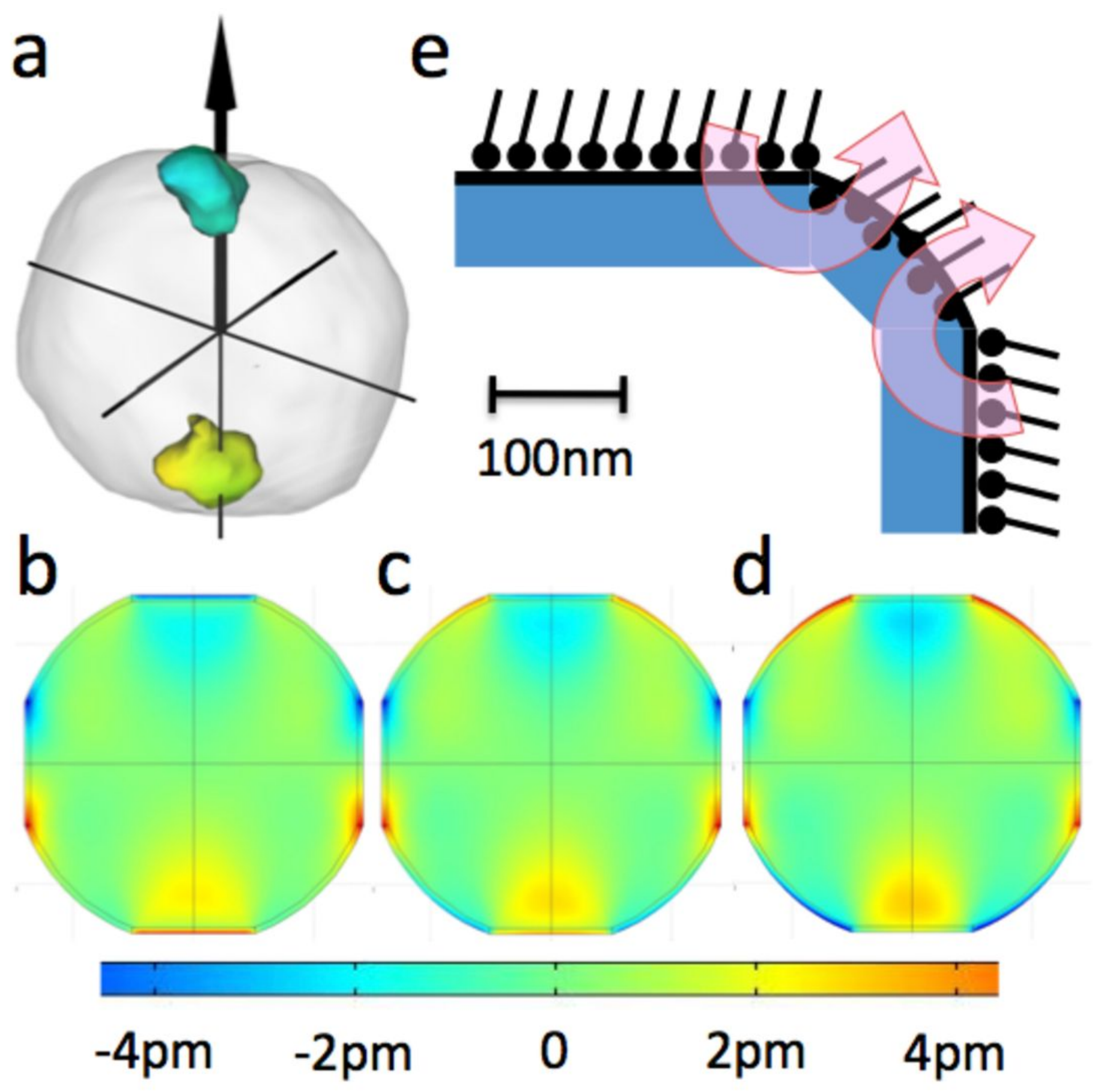

